\title{
The role of bicarbonate and base precursors in treatment of acute gastroenteritis
}

\author{
E J ELLIOTT, J A WALKER-SMITH, AND M J G FARTHING \\ Departments of Child Health and Gastroenterology, St Bartholomew's Hospital, London
}

\begin{abstract}
Widespread use of oral glucose-electrolyte rehydration solutions (ORS) has resulted in a dramatic decrease in the morbidity and mortality associated with childhood gastroenteritis, regardless of its aetiology. Nevertheless, diarrhoeal disease remains the most common cause of death of children in developing countries, and current research efforts are directed towards optimising the efficacy of ORS, aiming for both simplicity and economy. Controversy regarding the 'ideal' sodium and glucose concentration continues to the extent that different formulations are now recommended in the developing and industrialised world. The inclusion of bicarbonate or a base precursor (citrate, acetate, or lactate) in ORS is generally assumed to be necessary, both for promotion of water and sodium absorption and correction of acidosis. This issue, however, has received little critical attention since the formal introduction of oral rehydration therapy. We have reviewed the available evidence from animal and human studies and conclude that there is little evidence to support the inclusion of bicarbonate or a base precursor in ORS.
\end{abstract}

\section{Historical considerations}

Intravenous treatment. As early as 1832 Latta recommended intravenous saline containing 'two scruples of subcarbonate of soda' for the treatment of cholera and its associated acidosis. ${ }^{1}$ A similar regimen with intravenous sodium acetate or bicarbonate was used by 1910 by Sellards. ${ }^{2}$ This treatment decreased the mortality from renal failure associated with cholera. Acidosis in infantile diarrhoea of other causes was also shown to improve after administration of bicarbonate. ${ }^{3}$ Powers recommended bicarbonate in his plan of treatment for the 'intestinal intoxication of infants' in $1926,{ }^{4}$ and Hartmann used sodium lactate to relieve acidosis in diarrhoeal disease in $1938 .^{5}$ None of these studies, however, attempted to answer the important ques- tion as to whether rehydration with base was superior to rehydration with saline alone.

The use of parenteral bicarbonate was not without its problems. In the 1940s Rapoport et al recommended vigorous rehydration with saline containing sodium bicarbonate but without potassium and reported a 'post-acidotic state' in children whose diarrhoea had improved and in whom recovery seemed imminent. ${ }^{6}$ The clinical syndrome he described of lethargy, irritability, abnormal cardiac function, intracranial haemorrhage, generalised oedema, and tetany can be attributed to the alkalosis and electrolyte disturbance (hypokalaemia, hypocalcaemia, hypophosphataemia, and hypernatraemia) after excessive rapid administration of sodium and alkali.

Oral treatment. Darrow was the first to recommend oral rehydration with a glucose-electrolyte solution as an alternative to intravenous fluids. Although all the children in his original study had low serum bicarbonate concentrations, these returned to normal after treatment with solutions containing lactate $(53 \mathrm{mmol} / \mathrm{l})$, even in the most severe cases. Although this often took more than 24 hours, acidosis did not recur after rehydration began, and alkalosis and the 'post-acidotic state' were never observed. Further studies confirmed these findings, leading Darrow and colleagues to conclude that additional bases such as sodium bicarbonate were seldom if ever required for the treatment of acidosis in diarrhoeal disease of infancy. ${ }^{7}$ Furthermore, these investigators recognised that if bicarbonate was given rapidly or in large amounts the condition described by Rapoport could occur.

Since those early days, the use of oral rehydration solutions has rapidly increased. Bicarbonate or a base precursor is included in the World Health Organisation (WHO) ORS and in commercially manufactured ORSs in the United Kingdom (Table 1) and throughout the world, on the premises that 
Table 1 Composition of WHO and commercial ORSs (mmol/l)

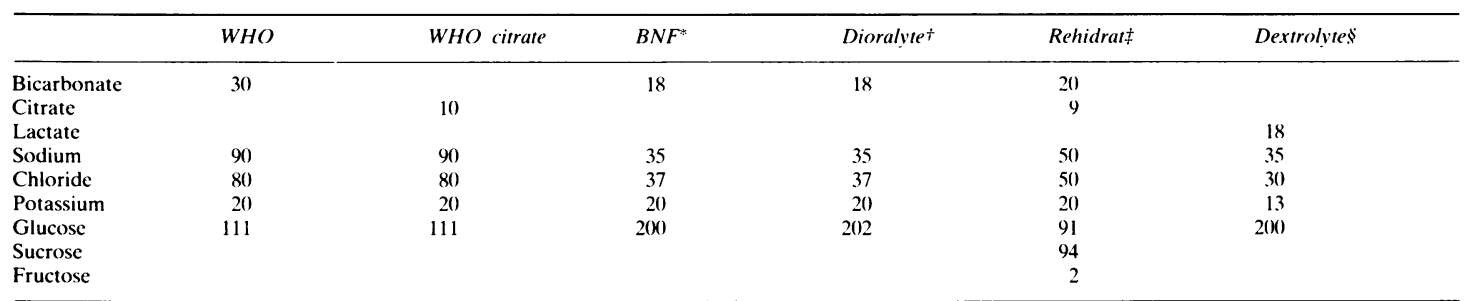

${ }^{*}$ British National Formulary, Sodium Chloride and Glucose Oral Powder Compound.

†Armour Pharmaceutical Co Ltd.

$\ddagger$ Searle Pharmaceuticals.

$\$$ Cow and Gate Ltd.

(a) bicarbonate enhances sodium and water absorption and (b) the metabolic acidosis of acute diarrhoea requires correction with exogenous base. Justification for the continued use of bicarbonate or base precursor in ORS must be supported by evidence for at least one or preferably both of these statements.

\section{Effect of base on intestinal sodium and water transport}

Bicarbonate stimulates water and sodium absorption in the normal human jejunum and ileum. ${ }^{8}$ The magnitude of this effect on sodium absorption in the human jejunum is comparable with that of glucose and independent of luminal $\mathrm{pH} .{ }^{9}$ Short chain fatty acids, such as the base precursor acetate, share the ability of bicarbonate to stimulate sodium and water absorption in the human small intestine. ${ }^{10}$

Although bicarbonate has similar effects on water and sodium absorption in the normal rat small intestine,${ }^{11}$ we have shown that bicarbonate and the base precursors acetate and citrate are unable (unlike glucose) to reverse water and sodium secretion induced by cholera toxin (Unpublished observations). These findings suggest that bicarbonate may not be beneficial with respect to the promotion of water absorption in the enterotoxin mediated diarrhoeas.

\section{Role of base in the correction of metabolic acidosis}

It is now generally accepted that in metabolic acidosis of childhood administration of bicarbonate should be reserved for conditions in which endogenous production or conservation of bicarbonate is inadequate to restore normal $\mathrm{pH}$. Examples of such specific disorders include salicylism, prolonged diarrhoea, disorders of organic and amino acid metabolism, and renal tubular acidosis. In acute hypoxia and prerenal or circulatory failure bicarbonate is only recommended when $\mathrm{pH}$ fails to correct after restoration of adequate intravascular volume. Similarly, children with diabetes with ketoacidosis do not require bicarbonate providing adequate insulin and fluids are given and cardiovascular and renal function is normal, unless blood $\mathrm{pH}$ falls below $7 \cdot 2 .{ }^{12}$

Metabolic acidosis during acute diarrhoea is generally attributed to bicarbonate loss in the stool, which results in loss of total blood buffering capacity. In addition, when dehydration is severe and renal plasma flow falls excretion of hydrogen ions and regeneration of bicarbonate is impaired. Also poor perfusion of peripheral tissue may contribute to a lactic acidosis. Acidosis is more likely to complicate cholera than other acute diarrhoea, as the stool concentration of bicarbonate is higher than in rotavirus or enterotoxigenic Escherichia coli diarrhoea and dehydration more pronounced (Table 2) ${ }^{13}{ }^{14}$ Despite these theoretical considerations and the common finding of a reduced serum bicarbonate concentration in acute diarrhoea, mean arterial $\mathrm{pH}$ is often normal.

Thus, providing perfusion is maintained, renal compensation ensures that acid base state will normalise with rehydration without additional base in the vast majority of cases of acute gastroenteritis.

In severely dehydrated or acidotic patients

Table 2 Electrolyte composition of stool in childhood gastroenteritis

\begin{tabular}{|c|c|c|c|c|}
\hline \multirow[t]{2}{*}{ Aetiology } & \multicolumn{4}{|c|}{ Stool electrolytes ( $\mathrm{mmol} / \mathrm{l})$} \\
\hline & Sodium & Potassium & Chloride & Bicarbonate \\
\hline \multirow[t]{2}{*}{ Cholera } & $101^{*}$ & 27 & 92 & 32 \\
\hline & $88+$ & 30 & 86 & 32 \\
\hline Rotavirus & $37 \dagger$ & 38 & 22 & 6 \\
\hline \multicolumn{5}{|l|}{ Enterotoxigenic } \\
\hline Escherichia coli & $53 \dagger$ & 37 & 24 & 18 \\
\hline Non-cholera diarrhoca & $56^{*}$ & 25 & 55 & 14 \\
\hline
\end{tabular}

*Reference 13 .

tReference 14 
$(\mathrm{pH}<7 \cdot 2)$, in those with persistent high volume diarrhoea, and in those with impaired renal function and worsening metabolic acidosis the administration of intravenous base such as sodium bicarbonate is indicated and may be life saving. Correction of acidosis with parenteral sodium bicarbonate must be performed with caution to avoid its detrimental effects. $^{12}$

\section{Clinical observations}

There is little clinical evidence to support the inclusion of base in the treatment of acidosis and dehydration in acute diarrhoea. Indeed, most regimens for intravenous rehydration are effective despite the omission of bicarbonate. ${ }^{15}$ In a controlled trial in Capetown Heese compared a mildly alkalinising intravenous solution $(26 \mathrm{mEq} / \mathrm{l}$ lactate) with or without additional sodium bicarbonate for treating children with acute gastroenteritis who were acidotic and $5-10 \%$ dehydrated. Those who received additional base regained normal $\mathrm{pH}$, carbon dioxide tension, and serum bicarbonate more quickly than those who did not, but no ill effects were suffered by the latter group, and both groups were biochemically identical by 24 hours. ${ }^{16}$

Early uncontrolled studies based in hospitals have shown equal effectiveness in rehydration but slower correction of acidosis when comparing a labon-gur (common salt and brown sugar) ORS without bicarbonate with the WHO solution. ${ }^{17}$ An uncontrolled study based in the community in the Punjab has shown successful treatment of acute diarrhoea in children and a substantial reduction in mortality even when both sodium bicarbonate and potassium chloride were omitted from the ORS. ${ }^{18}$ Similarly, rice water without electrolyte additives has been used successfully in the treatment of infantile gastroenteritis. ${ }^{19}$ In Cardiff 90 children admitted with gastroenteritis and reduced serum bicarbonate concentrations were adequately treated with intravenous or oral rehydration solutions without bicarbonate. $^{15}$

Controlled trials examining the value of base in ORS are few and are outlined in Table 3. In a recent clinical trial in Bangladesh Islam compared the efficacy of ORS with and without bicarbonate. ${ }^{20} \mathrm{He}$ showed that while correction of serum bicarbonate was slower in the children receiving the solution free of bicarbonate compared with the control group (WHO-ORS) during the first 24 hours, there was no difference at 48 hours. The delayed return of plasma bicarbonate to normal almost certainly has no clinical relevance as the patients were rehydrated, with good urine output and no clinical evidence of acidosis. A double blind controlled trial in the

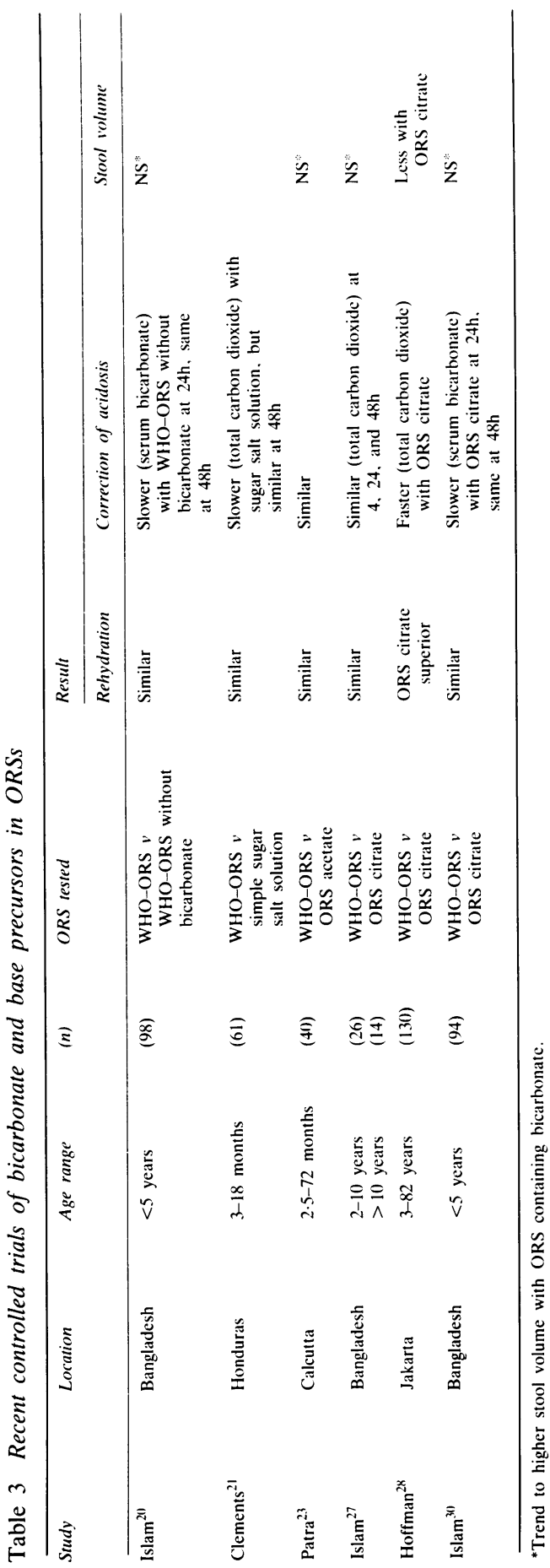


Honduras showed that a simple oral sugar/salt solution without bicarbonate will correct even pronounced acidosis (total carbon dioxide $<10 \mathrm{mmol} / \mathrm{l}$ ) as effectively, though slightly more slowly, than the WHO solution. ${ }^{21}$ We have recently completed a double blind controlled trial comparing British National Formulary ORS (Table 1) with an identical solution in which bicarbonate was replaced by chloride. In 40 children with gastroenteritis inclusion of bicarbonate offered no clinical or biochemical advantage (Unpublished observations).

\section{Practical problems with bicarbonate}

Although intravenous solutions containing bicarbonate have been widely used, they must be autoclaved under a high carbon dioxide pressure, making production of sterile solutions difficult. ${ }^{22}$ Regarding ORS, bicarbonate is often unavailable in developing countries and adds to the bulk and expense of ORS sachets. On exposure to high humidity or heat bicarbonate in ORS powders may form furfural compounds with glucose, causing decomposition of these substances, colouring the reconstituted solution brown, and making it less palatable.$^{23}$ Although this problem can be overcome by using chemically treated (encapsulated) sodium bicarbonate, this increases the cost of production. ${ }^{24}$ In aqueous solution bicarbonate may decompose to form carbonate. Additionally, flavouring agents, such as citric acid, used in some commercial formulations react with bicarbonate to form carbon dioxide and water with a resultant sharp fall in bicarbonate concentration in the solution. ${ }^{25}$ These difficulties associated with the use of bicarbonate have prompted the search for an alternative base.

\section{The use of base precursors}

The base precursors acetate, citrate, and lactate have been incorporated in intravenous and oral rehydrating fluids, ${ }^{22}$ although a possible drawback is that their metabolism may be seriously impaired in severe dehydration with circulatory compromise.

Controlled clinical trials of base precursors in ORS are outlined in Table 3. Patra, in a double blind controlled trial of infants with diarrhoeal dehydration and reduced plasma carbon dioxide concentrations (mean 14-15 mmol/l) found that ORSs containing acetate or bicarbonate were equally effective in correcting dehydration and acidosis but that patients were less willing to take solutions containing bicarbonate, which had discoloured brown..$^{2.3}$ Acetate salts, however, being highly hygroscopic, have a shorter shelf life than those containing sodium bicarbonate ${ }^{26}$ and turn to a jelly or gum like material by 12 hours at $37^{\circ} \mathrm{C}$ and more quickly if a small amount of water is added. Their use in powdered ORS, therefore, is not recommended.

In contrast, citrate is stable in tropical countries; no discoloration occurs during storage at up to $60^{\circ} \mathrm{C}$ for up to 3 years. ${ }^{24}$ Islam showed that an ORS containing tribasic sodium citrate compared favourably with the WHO ORS containing sodium bicarbonate, with respect to both its ability to rehydrate and to correct acidosis. ${ }^{27} \mathrm{~A}$ similar study in Jakarta concluded that the trisodium citrate based ORS resulted in less vomiting and stool output than the bicarbonate based ORS. Correction of serum carbon dioxide was more rapid with the citrate based ORS, despite a significantly lower serum carbon dioxide concentration on admission in the patients receiving this solution. ${ }^{28}$ Several other unpublished clinical trials conducted by the WHO diarrhoeal diseases control programme have compared citrate ORS and bicarbonate ORS. Although stool volume was higher when bicarbonate ORS was used in cholera, there was no difference in non-cholera diarrhoeas. ${ }^{29}$ Islam has recently investigated the use of tripotassium citrate monohydrate as a substitute for both sodium bicarbonate and potassium chloride in the WHO solution. ${ }^{30}$ The solution containing citrate resulted in slower correction of serum bicarbonate, but there was no difference between the groups at 48 hours.

Although several clinical studies suggest that citrate containing ORS may result in slightly lower stool volumes than equivalent bicarbonate containing ORS, the advantage of citrate over a solution without base has not been investigated.

It has been argued that the greater stability of lactate over citrate salts makes the former preferable for use in powdered ORS, but lactate containing solutions support mould growth, which may cause deterioration of glasswear and alter shelf life of the solution. ${ }^{21}$

\section{Conclusions}

When considering ORSs, particularly for use in developing countries, our prime concerns must be simplicity, effectiveness, and economy. Bicarbonate and base precursors (acetate and citrate) pose problems with respect to availability, cost, packaging, and stability. Further, they are of dubious benefit therapeutically, both for correction of acidosis and rehydration. Exclusion of these substances would decrease the production cost of prepackaged ORS, make preparation in the home easier, and possibly result in earlier beginning of oral rehydra- 
tion therapy, leading ultimately to the prevention of serious dehydration.

MJGF is a Wellcome Trust Senior Lecturer and gratefully acknowledges financial support by the Wellcome Trust.

\section{References}

' Latta T. Malignant cholera. Documents communicated by the Central Board of Health, London, relative to the treatment of cholera by the copious injection of aqueous and saline fluids into the veins. Lancet 1832;ii:274-7.

2 Sellards AW. Tolerance for alkalis in Asiatic cholera. Phillipine Journal of Science 1910;5:363-90.

${ }^{3}$ Howland J, McKim Marriott W. Acidosis occurring with diarrhea. Am J Dis Child 1916;11:309-25.

${ }_{4}$ Powers GF. A comprehensive plan of treatment for the so-called intestinal intoxication of infants. Am J Dis Child 1926;32: 232-57.

5 Hartmann AF, Perley AM, Basman J, Nelson MV, Asher C. Further observations on the metabolism and clinical uses of sodium lactate. J Pediatr 1938;10:692-723.

${ }^{6}$ Rapoport S, Dodd K, Clark M, Syllm I. Postacidotic state of infantile diarrhea: symptoms and chemical data. Am J Dis Child 1947;73:391-441.

7 Flett J, Pratt EL, Darrow DC. Methods used in treatment of diarrhea with potassium and sodium salts. Pediatrics 1949; 4:604-19.

* Turnberg LA, Bieberdorf FA, Morawski SG, Fordtran JS. Interrelationships of chloride, bicarbonate, sodium and hydrogen transport in the human ileum. J Clin Invest 1970;49:557-66.

9 Sladen GE, Dawson AM. Effect of bicarbonate on sodium absorption by the human jejunum. Nature 1968;218 (5138):267-8

10 Rolston DDK, Moriarty KJ, Farthing MJG, Kelly MJ, Clark ML, Dawson AM. Acetate and citrate stimulate water and sodium absorption in the human jejunum-implications for oral rehydration therapy. Digestion 1986;34:101-4.

"Mahalanabis D. Patra FC. In search of a super oral rehydration solution: can optimum use of organic solute-mediated sodium absorption lead to the development of an absorption promoting drug? Journal of Diarrheal Disease Research 1983;1:76-81.

12 Kappy MS, Morrow G. A diagnostic approach to metabolic acidosis in children. Pediatrics 1980;65:351-6.

13 Mahalanabis D, Wallace CK, Kallen RJ, Mondal A, Pierce NF. Water and electrolyte losses due to cholera in infants and small children: a recovery balance study. Pediatrics 1970;45:374-85.

${ }^{14}$ Molla AM, Rahman M, Sarker SA, Sack DA, Molla A. Stool electrolyte content and purging rates in diarrhoea caused by rotavirus, enterotoxigenic $\mathrm{E}$. coli and $\mathrm{V}$. cholerae in children. J Pediatr 1981;98:835-8.

is Price HV, Dodge JA, Thomas ME. Oral rehydration without added bicarbonate for childhood gastroenteritis. $\mathrm{Br} \mathrm{Med} \mathrm{J}$ 1984;289:532.

${ }^{16}$ Heese HV de, Tonin C, Bowie MD, Evans A. Management of metabolic acidosis in acute gastroenteritis. Br Med J 1966; ii: $144-6$.

17 Islam MR, Greenough III WB, Rahaman MM, Choudhury AKA, Sack DA. Labon-gur (common salt and brown sugar) oral rehydration solution in the treatment of diarrhoea in adults. J Trop Med Hyg 1980;83:41-5.

${ }^{18}$ Kielmann AA, McCord C. Home treatment of childhood diarrhea in Punjab villages. J Trop Pediatr 1977;23:197-201.

19 Wong HB. Rice water in treatment of infantile gastroenteritis. Lancet 1981;ii:102-3.

21) Islam MR, Ahmed SM. Oral rehydration solution without bicarbonate. Arch Dis Child 1984;59:1072-5.

21 Clements ML, Levine MM, Cleaves F, et al. Comparison of simple sugar/salt versus glucose/electrolyte oral rehydration solutions in infant diarrhoea. J Trop Med Hyg 1981;84:189-94.

22 Cash RA, Nalin DR, Khondakar MMT, Huq Z, Phillips RA. Acetate in the correction of acidosis secondary to diarrhoea. Lancet 1969;ii:302-3.

23 Patra FC, Mahalanabis D, Jalan KN, Sen A, Benerjee P. Can acetate replace bicarbonate in oral rehydration solution for infantile diarrhoea? Arch Dis Child 1982;57:625-7.

24 World Health Organisation/United Nations International Children's Emergency Fund. Oral rehydration salts. Planning, establishment and operation of production facilities. $\mathrm{WHO} /$ $C D D / S E R \quad 1985 ; 85: 1-136$.

25 Rolston DDK, Borodo MM, Farthing MJG. Bicarbonate loss from commercial oral rehydration solution. Lancet 1985;i: 638-9.

${ }^{26}$ De Cespedes C. Oral rehydration salts have a shorter shelf life with sodium acetate than with sodium bicarbonate. Arch Dis Child 1983;58:234-5.

27 Islam MR, Samadi AR, Ahmed SM, Bardhan PK, Ali A. Oral rehydration therapy: efficacy of sodium citrate equals to sodium bicarbonate for correction of acidosis in diarrhoea. Gut 1984;25:900-4.

${ }^{28}$ Hoffman SL, Moechtar MA, Simantuntak CH, et al. Rehydration and maintenance therapy of cholera patients in Jakarta: citrate-based versus bicarbonate-based oral rehydration salt solution. J Infect Dis 1985;152:1159-65.

${ }^{29}$ World Health Organisation Diarrhoeal Diseases Control Programme. Oral rehydration salts (ORS) formulation containing trisodium citrate. WHO/CDD/SER 1984;7.

3) Islam MR. Can potassium citrate replace sodium bicarbonate and potassium chloride of oral rehydration solution? Arch Dis Child 1985;60:852-5.

Correspondence to Dr M J G Farthing, Department of Gastroenterology, St Bartholomew's Hospital, West Smithfield, London EC1A 7BE. 